\title{
Hydroxyurea Regressed Atherosclerosis Plaques in ApoE-/- mice: A Discovery Based on Clinic
}

\section{Qian Tong}

Jilin University First Hospital

\section{Xinyu Yang}

Jilin University First Hospital

\section{Shu-Rong Ma}

Chinese Academy of Medical Sciences \& Peking Union Medical College Institute of Materia Medica

Xian-Feng Zhang

Jilin University First Hospital

Jian-Dong Jiang

Chinese Academy of Medical Sciences \& Peking Union Medical College Institute of Materia Medica

Yan Wang ( $\nabla$ wangyan@imm.ac.cn )

Chinese Academy of Medical Sciences \& Peking Union Medical College Institute of Materia Medica https://orcid.org/0000-0001-7924-4988

\section{Research article}

Keywords: Hydroxyurea (HU), Atherosclerosis, Clinical based, ApoE-/- mice, Pharmacodynamics

Posted Date: September 17th, 2020

DOI: https://doi.org/10.21203/rs.3.rs-66809/v1

License: (c) (1) This work is licensed under a Creative Commons Attribution 4.0 International License.

Read Full License 


\section{Abstract}

Background: A 58-year-old lady was introduced to the clinic because of acute coronary syndrome combined with essential thrombocythemia. After treating her with aspirin, statins and hydroxyurea (HU), the plaques in her coronary arteries showed improvement dramatically. Here, we aim to investigate that whether $\mathrm{HU}$ reduces atherosclerosis plaques in $\mathrm{ApoE}^{-/-}$mice.

Methods: Wild-type (C57BL/6, $\mathrm{n}=8$ ) and Apolipoprotein E knockout (ApoE-/-, $\mathrm{n}=40$ ) mice were used in atherosclerosis model and medication groups. The mice were separated into 7 groups, including the normal control group, the atherosclerosis model group, the dual antiplatelet therapy group (aspirin, and clopidogrel bisulfate), the low-dose and high-dose HU therapy groups [aspirin, clopidogrel bisulfate, and $\mathrm{HU}(10$ or $20 \mathrm{mg} / \mathrm{kg} /$ day)], the positive medicine group (aspirin, clopidogrel bisulfate, and atorvastatin calcium), and the combined medicine treatment group [aspirin, clopidogrel bisulfate, atorvastatin calcium, and $\mathrm{HU}(10 \mathrm{mg} / \mathrm{kg} /$ day)]. Fasting serum and aortic vessels were obtained after experiment. The aortic oil red $\mathrm{O}$, hematoxylin-eosin $(\mathrm{H} \& \mathrm{E})$, and full-length oil red $\mathrm{O}$ staining were performed to evaluate the HU's efficacy of anti- atherosclerosis, and the blood lipid / glucose levels and liver / kidney functions were evaluated after HU treatment.

Results: The oil red $\mathrm{O}$ and $\mathrm{H} \& \mathrm{E}$ staining results came out that $\mathrm{HU}$ therapy with antiplatelet showed an obvious effect in decreasing atherosclerosis plaques and the effect of HU therapy (10 or $20 \mathrm{mg} / \mathrm{kg}$ ) was stronger than dual antiplatelet therapy plus statin, without liver and kidney toxicity observed. Furthermore, the combined drugs with $\mathrm{HU}(10 \mathrm{mg} / \mathrm{kg} /$ day $)$, statin and antiplatelet nearly eliminated the plaques.

Conclusions: A discovery based on clinic reveals that $\mathrm{HU}$ regressed atherosclerosis plaques in ApoE-/mice, which provides us a new insight into anti-atherosclerosis drugs strategy.

\section{Background}

Atherosclerosis disease is the most common chronic disease all over the world [1].Now it is considered to be a complex metabolic disorder, with major risk factors including high blood pressure, high blood lipids and heavy smoking, diabetes, obesity and genetic factors [2]. During the development of atherosclerosis, intimal injury, inflammatory response, oxidative stress, lipid infiltration, platelet activation, and vascular smooth muscle cell activation may occur ${ }^{1}$. Despite the complicated pathogenesis, oral drug therapy for atherosclerosis mainly focuses on the areas of anti-platelet, regulating lipid metabolism and improving endothelial function currently [3].

Anti-platelet therapy is the most important therapy in the treatment of atherosclerosis. It prevents cardiovascular events by inhibiting platelet aggregation and adhesion. Numerous clinical trials and basic studies have demonstrated the effectiveness of such therapy [4]. However, side effects of such drugs include that the drug reactivity of individuals varies greatly and the increased risk of bleeding in patients have not been effectively addressed [5]. Other essential drugs for treating atherosclerosis are lipid-lowing drugs, among which the mainstream drugs include HMG-CoA reductase inhibitors, such as statins [6] and 
fibrates [7] that enhance high density lipoprotein (HDL) synthesis. These drugs inhibit the development of atherosclerosis by improving the lipid profile. In addition, statins can reduce the incidence of atherosclerosis by improving endothelial function and stabilizing atherosclerotic plaques [8]. However, the renal toxicity, hepatotoxicity, myotoxicity [9] and new-onset diabetes [10] increased its risk of long-term use.

In recent years, it has been proven that inflammatory response plays an important role in the occurrence and development of atherosclerosis and participates in the formation, and progression of plaques [1113]. Also, attention has been focused on anti-tumor approaches in treating atherosclerosis through antiinflammatory pathways, and some new therapeutic targets have been identified [14]. Hydroxyurea (HU) is a non-alkylated anti-tumor and anti-viral drug that has been widely used in clinical practice. It has been reported to be a nucleoside diphosphate reductase inhibitor, which can block the reduction of nucleotides to deoxynucleotides, interfere with purine and pyrimidine base biosynthesis, and selectively block DNA synthesis. Even though it can be synthesized artificially since 1869 [15], it is a first-line anti-tumor drug for several malignant diseases currently [16]. In 2009, Gallaugher has reported that high dose of hydroxyurea $(400 \mathrm{mg} / \mathrm{kg})$ reduced the degree of aortic atherosclerosis in secondary injury rabbit model of atherosclerosis [17]. However, the efficacy and mechanism of HU in atherosclerosis have not been investigated. Here, we reported a discovery based on clinic that hydroxyurea regressed atherosclerosis plaques in apolipoprotein $\mathrm{E}$ knockout $\left(\mathrm{ApoE}^{-/-}\right)$mice.

\section{Methods}

\section{Clinical case presentation- acute coronary syndrome (ACS) combined with ET}

A 58-year-old woman was admitted to the hospital because of sustaining chest pain for 8 hours, she had a history of stable angina for 5 years and was diagnosed essential thrombocythemia 2 years ago, but she didn't receive any treatment in this duration. Biochemical examination showed blood platelet level at 1140×1012/L (normal range at 125-350×1012/L), and myocardial injury markers and other biomarkers were at normal ranges. Electrocardiogram showed inverted T wave in V1-V6 Leads. The patient was diagnosed ACS and underwent a coronary angiography (CAG) as soon as admitted, result showed a severe diffuse stenosis in proximal left anterior descending coronary artery (LAD) and moderate stenosis in distal right coronary artery (RCA) (Fig. 1). After consultations with hematologists and cardiac surgeon, the patient underwent coronary artery bypass grafting (CABG).

After the surgery, she started oral medical treatment of ET and ACS using aspirin $100 \mathrm{mg}$ QD, atorvastatin 20mg QD and hydroxyurea 100mg BID and have herself a reexamination once in a while.

The data and information of the case was approved by the institution ethnic committee of the First Hospital of the Jilin University and was published with the patients' consent.

\section{Chemicals and Reagents}


Atorvastatin calcium, aspirin, hydroxyurea (purity> 98\%) for animals were purchased from Solarbio Biotechnology, Co., Ltd. (Beijing, China). Clopidogrel bisulfate (purity $>98 \%$ ) was provided by the $\mathrm{TCl}$ chemical industry development co., Ltd (Shanghai, China). Berberine (98\%) was obtained from J\&K scientific Co., Ltd. The saturated oil red O solution, OCT frozen section embedding agent, $4 \%$ tissue cell fixation solution and hematoxylin dye solution were purchased from Solarbio Biotechnology. Co., Ltd (Beijing, China). Deionized distilled water was obtained from Hangzhou Wahaha Group Co. Ltd.

(Hangzhou, China). Chromatography grade methanol and isopropanol were obtained from Thermo Fisher Scientific, Co., Ltd. (Fair Lawn, NJ, United States). Other chromatographic reagents were obtained from Sinopharm Chemical Reagent Co., Ltd. (Beijing, China). Serum glucose (Glu), triglycerides (TG), total cholesterol (CHO) and low-density lipoprotein cholesterol (LDL-C) kits were purchased from Biosino BioTechnology \& Science Inc. Alanine aminotransferase (ALT), aspartate aminotransferase (AST), and creatinine kits were obtained from Nanjing Jiancheng Bioengineering Institute.

\section{Animals}

Eight 8-Week old wild-type (C57BL/6) and forty eight Apolipoprotein E knockout (ApoE ${ }^{-/}$) mice (8 weeks) were acquired from Beijing Vital River Laboratory Animal Technology Co., Ltd. The animals were housed in the SPF-grade rooms with a temperature of $22-24{ }^{\circ} \mathrm{C}$, a humidity of $45 \%$, and a 12 -h light/dark cycle (light time from 8:00 to 20:00). All the mice had free access to food and water during the treatment. All experiments were conducted in accordance with institutional and ethics guidelines and were approved by the Laboratories Institutional Animal Care and Use Committee of the Chinese Academy of Medical Sciences and Peking Union Medical College (No. 00001020).

\section{Grouping and medication}

The grouping rules were as following: wild-type C57 mice were used in normal control groups and $\mathrm{ApoE}^{-1-}$ mice were used in atherosclerosis model and medication groups. After acclimatization with normal diet for a week, the model groups and medication groups were fed with high-fat diet (HFD: $21 \%$ fat, $0.15 \%$ cholesterol and $78.85 \%$ regular diet) for 4 weeks, then, all the groups were treated with saline or drugs for 8 weeks for efficacy study. The normal control groups were fed with normal diet. Fasting serum were obtained after treatment. Then, the animals were sacrificed by cervical dislocation and aortic vessels were obtained for experiment. Among them, six aortic vessels in each group were preserved for pathological staining and the rests were preserved for aortic full-length staining.

All the mice were separated into 7 groups $(n=8)$ : (1) The normal control group: normal diet and normal saline (oral, $0.2 \mathrm{~mL}$ ); (2) Atherosclerosis model group: high-fat diet and normal saline (oral, $0.2 \mathrm{~mL}$ ); (3) Dual antiplatelet therapy group: high-fat diet, aspirin (oral, $5 \mathrm{mg} / \mathrm{kg} / \mathrm{day}$ ), and clopidogrel bisulfate (oral, $25 \mathrm{mg} / \mathrm{kg} /$ day); (4) Low-dose HU therapy group: high-fat diet, aspirin (oral, $5 \mathrm{mg} / \mathrm{kg} /$ day), clopidogrel bisulfate (oral, $25 \mathrm{mg} / \mathrm{kg} /$ day), and HU (oral, $10 \mathrm{mg} / \mathrm{kg} /$ day); (5) High-dose HU therapy group: high-fat diet, aspirin (oral, $5 \mathrm{mg} / \mathrm{kg} /$ day), clopidogrel bisulfate (oral, $25 \mathrm{mg} / \mathrm{kg} / \mathrm{day}$ ), HU (oral, 20 $\mathrm{mg} / \mathrm{kg} /$ day); (6) Positive medicine group: high-fat diet, aspirin (oral, $5 \mathrm{mg} / \mathrm{kg} /$ day), clopidogrel bisulfate 
(oral, $25 \mathrm{mg} / \mathrm{kg} / \mathrm{day}$ ), and atorvastatin calcium (oral, $5 \mathrm{mg} / \mathrm{kg} /$ day); (7) Combined medicine treatment group: high-fat diet, aspirin (oral, $5 \mathrm{mg} / \mathrm{kg} /$ day), clopidogrel bisulfate (oral, $25 \mathrm{mg} / \mathrm{kg} /$ day), atorvastatin calcium ( $5 \mathrm{mg} / \mathrm{kg} /$ day), and $\mathrm{HU}$ (oral, $10 \mathrm{mg} / \mathrm{kg} /$ day).

\section{Oil red 0 staining}

The proximal aorta that attached the heart was used for cross-sections. The collected aortic tissue was fixed in $4 \%$ paraformaldehyde for 24 hours, and dehydrated overnight. Then, the tissue was embedded in the embedding agent OCT to prepare frozen slices $(5 \mu \mathrm{m})$ under $-20^{\circ} \mathrm{C}$. Saturated oil red $\mathrm{O}$ solution that dissolved in isopropanol was mixed with distilled water by a ratio of 3: $2(\mathrm{~V}: \mathrm{V})$. After filtration, the frozen slices were stained in the diluted oil red 0 working solution at room temperature for 6 hours. Then, rinse the slices in $60 \%$ isopropanol-water solution for 3 times and once in running water. The slices were restrained in hematoxylin solution for 1-2 min and washed by running water. The dyed slices were sealed by neutral gelatin, photographed and recorded by microscope system (Leica, Germany).

\section{Hematoxylin-eosin (H \& E) staining}

The aortic tissue was fixed in $4 \%$ paraformaldehyde for 24 hours after collected and dehydrated for 16 hours by an automatic dehydrator. After routine embedded with paraffin in automatic embedding machine, cut the tissue into sections $(5 \mu \mathrm{m})$. Then, place the sections in an oven $\left(60^{\circ} \mathrm{C}\right)$ for 1 hours and dewax by xylene, ethanol and water. The sections were further stained by hematoxylin for 10 minutes and placed under running water to remove residual color. $5 \%$ acetic acid was used to differentiate, and then stained by eosin. Finally, seal the sections with neutral gelatin after dried and captured by the microscope system. The corrected plaque areas were calculated by $\mathbb{\text { Area }}$ of plaques / Area of vascular $\times 100 \%$ in the corresponding cross section. The statistical results of corrected plaque area were the average values of the plaque area obtained by the oil red $\mathrm{O}$ staining and H\&E staining of each mouse.

\section{Full length staining of aorta by oil red 0}

The aortic tissue that attached the heart of the animals were removed after sacrifice, and dehydrated in a $20 \%$ sucrose solution overnight. After rinsed with PBS solution, remove the excess tissue on the aorta under the microscope. The tissue was stained for 6 hours at room temperature in oil red 0 working solution. Then, the plaques were stained red. After that, the stained aorta was taken out, and rinsed for 3 times in $60 \%$ isopropanol-water. Soak the tissue in PBS, cut the blood vessel longitudinally and pin it flat on a black silicone pad. The image of the aorta tissue was record by camera (Canon EOS 700D, New York, USA).

\section{Serum and Plasma analysis}

Serum and plasma samples were collected from fundus venous plexus and the tubes for collecting plasma were pretreated by heparin sodium. Serum glucose (Glu), triglycerides (TG), total cholesterol $(\mathrm{CHO})$ and low-density lipoprotein cholesterol (LDL-C) were measured according to the instructions of kit. 
Levels of alanine aminotransferase (ALT), aspartate aminotransferase (AST), and creatinine in plasma were determined by test kits and the experiment steps referred to the instructions.

\section{Statistical analysis}

All the data were analyzed by Graphpad Prism 5.0 version and presented as the means \pm standard deviation (SD). The atatistical analyses were conducted using two-way Student's t-test and $P$ values less than 0.05 were considered statistically significant.

\section{Results}

Clinical case outcome - HU may have a positive effect on decreasing atherosclerosis plaques with thrombocythemia (ET)

Symptom of angina and dyspnea had relieved once after coronary artery bypass grafting (CABG) surgery. According to the follow-up, count of platelets (PLT) of patient had reduced significantly (Table 1). After 10 months' treatment, the dose of hydroxyurea was reduced to $100 \mathrm{mg}$ once a day (OD). The patient didn't occur any type of angina for 3 years after CABG, and didn't evaluate the condition of coronary artery nor bypass vessel. During the latest re-examination, we ran a computer tomography angiography (CTA) on coronary artery for the patient and result came out that no lesion on coronary was found. Meanwhile, bypass vessel was not visualized. Coronary angiography (CAG) and intravascular ultrasound (IVUS) exam were performed. Compared to the result of the first admission when severe stenosis of LAD coronary artery (in Fig. 1a) and moderate stenosis in right coronary artery (RCA, in Fig. 1b) were found, it came out that only mild circular lesion in proximal LAD (in Fig. 2a ( $L, C, R)$ ) and RCA (in Fig. $2 b(L, C, R)$ ).

Table 1

Level of PLT during oral medication therapy

\begin{tabular}{|lllllll|}
\hline Months after OMT & 3 & 6 & 9 & 12 & 24 & 36 \\
\hline Level of PLT (109/L) & 374 & 421 & 477 & 254 & 314 & 342 \\
\hline
\end{tabular}

\section{Hu Decreases Atherosclerosis Plaque Areas In Apoe Mice}

ApoE knockout mice (ApoE ${ }^{-/-}$mice) are the most widely used animal models in evaluating atherosclerotic drugs [18]. Herein, to further evaluate the anti-atherosclerotic effects of the combined therapy of $\mathrm{HU}$ and anticoagulants, the high-fat diet induced atherosclerotic model in $\mathrm{ApoE}^{-/-}$mice were used for pharmacodynamics studies.

According to the aortic oil red O (Fig. 3a), H\&E (Fig. 3b), and full-length oil red O (Fig. 3c) staining, the aortic vessels in the atherosclerosis model group presented large area of lipid-containing lesions and obvious plaques than the normal control group after 12 weeks of modeling, which indicated the success of modeling. In the dual antiplatelet therapy group, corrected area of atherosclerotic plaques significantly 
decreased by comparison with the model group, by $52 \%$ as shown in Fig. $3 d\left(n=6,{ }^{* \star} P<0.01\right)$. After 8 weeks of additional HU therapy, the areas of plaques showed a further significant decrease, by $76 \%$ and $85 \%$ in the both HU groups (low-dose and high-dose) with $P$ values less than 0.001 , when in comparison with the model group (Fig. 3d). Moreover, the result suggested that the anti-atherosclerotic effects of $\mathrm{HU}$ addition (high-dose) has largely improved when compared with the dual antiplatelet therapy (-85\% vs. $-52 \%, \star P<0.05$ in Fig. 3d). Additionally, the efficacy of the high dose HU group was more obvious than the combined drugs of antiplatelet and atorvastatin (the positive medicine group), with a reduction of $-85 \%$ and $-64 \%$, respectively (in Fig. 3d). Again, after addition of $\mathrm{HU}$, the combined medicine group (drugs of antiplatelet and atorvastatin) with $\mathrm{HU}$ (low-dose, $10 \mathrm{mg} / \mathrm{kg} / \mathrm{d}$ ), presented a further decrease of the number and area of aortic plaques which were nearly eliminated $\left(-91 \%,{ }^{* \star *} P<0.001\right.$, compared with the model group, in Fig. 3d), indicating that $\mathrm{HU}$ addition performed a satisfying effect on atherosclerosis after combined with drugs of antiplatelet and atorvastatin.

\section{$\mathrm{HU}$ slightly decreased the lipid/glucose levels in $\mathrm{ApoE}^{-/-}$mice without significance}

Serum glucose (Glu), total cholesterol (CHO), low density lipid cholesterol (LDL-C) and triglyceride (TG) levels are important indicators to evaluate the risk of atherosclerosis. As shown in Fig. 4, all of those indicators were significantly elevated than those in normal control group, proving the disorder of metabolism in glucose and lipids in the model group. Among all the HU administrated groups, Glu (Fig. 4a), TG (Fig. 4b), CHO (Fig. 4c), LDL-C (Fig. 4d) and levels were significantly lower than that of model group $(n=8)$, which means that after drugs treatment, the metabolism of glucose and lipids had improved in the mice model, but with no significance with low-dose and high-dose treatment groups of HU. In addition, by comparison with the dual antiplatelet group, we didn't see a prominent change in terms of aforementioned indicators, the glucose and lipid levels of the dual antiplatelet group (dual antiplatelet group vs. high-dose HU group) are shown as following: $\mathrm{Glu}(\mathrm{mmol} / \mathrm{L}), 1.019 \pm 0.021$ vs. $1.022 \pm 0.050$; $\mathrm{CHO}$ (mmol/L), $5.561 \pm 1.266$ vs. $5.134 \pm 0.589 ; \mathrm{LDL}-\mathrm{C}$ (mmol/L), $2.350 \pm 0.631$ vs. $2.199 \pm 0.411 ; \mathrm{TG}$ $(\mathrm{mmol} / \mathrm{L}), 2.886 \pm 0.308 \mathrm{vs} .3 .025 \pm 0.1573$. Apart from this, $\mathrm{HU}$ addition did not obviously lower the blood glucose and TG levels in the combined medicine group, while showed a decrease trend in $\mathrm{CHO}$ and LDL-C levels [CHO (mmol/L), $5.186 \pm 1.044$ vs $4.118 \pm 1.121$; LDL-C (mmol/L), $2.207 \pm 0.568$ vs $1.791 \pm$ 0.490]. This might suggest that the pharmacodynamic mechanisms of $\mathrm{HU}$ in anti-atherosclerosis might not be mainly through the improvement of glucolipid dysfunction, and other possible mechanisms could be explored.

\section{Hu Showed Good Safety In Liver And Kidney Function}

Levels of alanine aminotransferase (ALT), aspartate aminotransferase (AST), and creatinine in plasma are important indicators of evaluating liver and kidney functions. Herein, these indexes were determined by kits after 8 week's treatment in the animal model. As shown in Fig. 5, there were no significant changes in terms of AST (Fig. 5a) and ALT (Fig. 5b) activities in the low-dose and high-dose HU therapy group ( $\mathrm{n}=$ 8), by comparison of the dual antiplatelet therapy group. The results showed that liver toxicity was not 
observed after administration of $\mathrm{HU}(10$ or $20 \mathrm{mg} / \mathrm{kg} / \mathrm{d}$ ). While, in the positive medicine group (antiplatelet and atorvastatin), both enzymes showed the increasing trends (by $72 \%$ in AST and $20 \%$ in ALT); when combined with HU (antiplatelet, atorvastatin and HU), AST and ALT increased to $80 \%$ and $40 \%$. Furthermore, the addition of $\mathrm{HU}$ did not increase the creatinine content when compared with the double-antiplatelet treatment group (Fig. 5c), suggesting that neither significant hepatotoxicity nor renal toxicity occurred after treatment with $\mathrm{HU}$ and antiplatelet drugs (aspirin $5 \mathrm{mg} / \mathrm{kg} / \mathrm{d}$, clopidogrel bisulfate $25 \mathrm{mg} / \mathrm{kg} / \mathrm{d}$, HU 10 or $20 \mathrm{mg} / \mathrm{kg} / \mathrm{d}$ ).

\section{Discussion}

As mentioned, all of the mainstream anti-atherosclerosis drugs have significant side effects, such as the risk of bleeding caused by anti-platelet drugs, rhabdomyolysis [19] and new on-set diabetes caused by statins. There is still a lackage of effective and safe anti-atherosclerosis drugs.

Currently, anti-tumor drugs such as rapamycin is widely used as the coating of drug eluting stent (DES) and drug coating balloon (DCB) for coronary artery intervention. The anti-tumor mechanism of rapamycin includes inhibition of vascular endothelial growth factor-induced endothelial proliferation [20] and preventing the cell from making DNA replication and normal cell division [21], which may explain the mechanism of treating atherosclerosis. Rapamycin is usually eluted on the polymer coating of stent and released slowly during the healing period of coronary intervention. Compare to the bare metal stent, clinical studies have shown that rapamycin drug-eluting stents have a lower restenosis rate than patients with bare metal stents, thereby reducing the probability of recurrence of myocardial infarction [22]. Other anti-tumor drugs such as methotrexate [23, 24], sulfur purine [25] are also reported to be effective for atherosclerosis. However, the role of anti-tumor drugs in atherosclerosis is limited to DES and DCB, no oral anti-tumor drugs are approved to be put in clinical use toward atherosclerosis.

In our study, we treated an aged lady of both ACS and ET using HU in combination with aspirin and statins. After 3-year's follow-up, the atherosclerosis plaque has significantly digressed, which may suggest that $\mathrm{HU}$ could inhibit atherosclerotic stenosis of vascular arteries. In animal experiments, we found that the lower dose $(10,20 \mathrm{mg} / \mathrm{kg})$ of HU combined with dual-antiplatelet therapy and statin has been preliminarily proved to be able to significantly reduce atherosclerotic plaque area and the effect is superior to dual-antiplatelet therapy. Furthermore, the drug effect is obviously dose-dependent, prompting the possible effect of $\mathrm{HU}$ on atherosclerosis.

However, compared with the dual-antiplatelet group, reduction of blood glucose and lipid levels was not obvious, suggesting that the pharmacodynamic mechanism of HU's anti-atherosclerosis effect may not be mainly through the improvement of glucose-lipid dysfunction. The pharmacodynamic mechanism and pharmacokinetics of its anti-atherosclerosis effect need further study.

Meanwhile, there were no significant changes in serum ALT, AST and creatinine in the HU treatment group compared with the dual-antiplatelet treatment group, suggesting that no significant hepatotoxicity or renal toxicity occurred after $\mathrm{HU}$ treatment at this effective dose. 


\section{Conclusions}

The complex mechanisms of atherosclerosis have limited the efficacy of marketed drugs all the time, and the innovative and effective drugs or combined therapies are necessary. We accidentally found the potential of $\mathrm{HU}$ in anti-atherosclerosis during the clinical treatment to prevent atherosclerosis, and proved its efficacy and safety through animal experiments. This study might provide a new insight into antiatherosclerosis drugs.

\section{Abbreviations}

ACS: acute coronary syndrome; $\mathrm{ApoE}^{-/}$: apolipoprotein E knockout; ALT: alanine aminotransferase; AST: aspartate aminotransferase; $\mathrm{CABG}$ : coronary artery bypass grafting; CAG: coronary angiography; $\mathrm{CHO}$ : total cholesterol; CTA: computer tomography angiography; DCB: drug coating balloon; DES: drug eluting stent; ESM: electronic supplementary material; ET: essential thrombocythemia; Glu: serum glucose; $\mathrm{HU}$ : hydroxyurea; HDL: high density lipoprotein; IVUS: intravascular ultrasound; LAD: left anterior descending coronary artery; LDL-C: low-density lipoprotein cholesterol; RCA: right coronary artery; SD: standard deviation; TG: triglycerides.

\section{Declarations}

\section{Authors' Contributions}

Q. T. contributed to conceptualization, methodology, investigation, writing - original draft preparation. X. Y. Y. performed resources, writing the original draft preparation, and analyzed the data. S. R. M. contributed to formal analysis, software, visualization, validation, writing / review/editing. J. D. J. and Y. W. conceived of conceptualization, project administration, supervision, writing / review / editing. All authors have read and approved the submission of the manuscript. All authors have agreed to authorship and order of authorship for this manuscript and all authors have the appropriate permissions and rights to the reported data.

\section{Funding}

The project was supported by the National Natural Science Foundation of China (Nos. 81573493 and 81973290), CAMS Innovation Fund for Medical Sciences (CIFMS, No. 2016-I2M-3-011, China), Beijing Key Laboratory of Non-Clinical Drug Metabolism and PK/ PD study (Z141102004414062, China), the National Megaproject for Innovative Drugs (Nos. 2018ZX09711001-002-002 and 2018ZX09302015, China), and Beijing Natural Sciences Fund Key Projects (NO. 7181007).

\section{Acknowledgements}

We thank Shimadzu (China) Co., Ltd. for the technological supports in this study. 
All procedures performed in studies involving human participants were in accordance with the ethical standards of the institution ethnic committee of the First Hospital of the Jilin University and with the 1964 Helsinki Declaration and its later amendments or comparable ethical standards. This is an observational study and the institution ethnic committee of the First Hospital of the Jilin University has confirmed that no ethical approval is required. The data and information of the clinical case was published with the patients' written consent.

Animal experiments were conducted in accordance with institutional and ethics guidelines and were approved by the Laboratories Institutional Animal Care and Use Committee of the Chinese Academy of Medical Sciences and Peking Union Medical College (No. 00001020).

\section{Consent for publication}

The authors declared that they had obtained written informed consent for publication of this manuscript and the personal details from this individual.

\section{Competing interest}

The authors declared that they had no competing interests.

\section{Availability of data and materials}

The data used and/or analyzed during the current study are available from the corresponding author on reasonable request.

\section{References}

1. Roth GA, Johnson C, Abajobir A, et al. Global, regional, and national burden of cardiovascular diseases for 10 causes, 1990 to 2015. J Am Coll Cardiol. 2017;70(1):1-25.

2. Haberka M. Skilton M, Biedroń M, et al. Obesity, visceral adiposity and carotid atherosclerosis. J Diabetes Complications. 2019;33(4):302-6.

3. Fleg JL. Forman DE, Berra K, et al. Secondary prevention of atherosclerotic cardiovascular disease in older adults: a scientific statement from the American Heart Association. Circulation. 2013;128(22):2422-46.

4. Silber S. Secondary prevention after myocardial infarction with prolonged dual anti-platelet therapy (DAPT). A new therapeutic concept. MMW Fortschr Med. 2017;159:49-52.

5. Grodzinsky A. Arnold SV, Wang TY, et al. Bleeding risk following percutaneous coronary intervention in patients with diabetes prescribed dual anti-platelet therapy. Am Heart J. 2016;182:111-8.

6. Deckers JW. Blumenthal RS. Statins for primary prevention of cardiovascular disease. BMJ. 2011;342:d1048. 
7. Pavanello C. Baragetti A, Branchi A, et al. Treatment with fibrates is associated with higher $L A L$ activity in dyslipidemic patients. Pharmacol Res. 2019;147:104362.

8. Puri R. Libby P, Nissen SE, et al. Long-term effects of maximally intensive statin therapy on changes in coronary atheroma composition: insights from SATURN. Eur Heart J Cardiovasc Imaging. 2014;15(4):380-8.

9. Ward NC. Watts GF, Eckel RH. Statin Toxicity: mechanistic insights and clinical implications. Circ Res. 2019;124:328-50.

10. Montastruc F, Benevent J, Rousseau V, et al. Risk of diabetes with fibrates and statins: a pharmacoepidemiological study in Vigibase. Fundam Clin Pharmacol..

11. Bobryshev YV. Nikiforov NG, Elizova NV, Orekhov AN. Marcophages and their contribution to the development of atherosclerosis. Results Probl Cell Differ. 2017;62:273-98.

12. Serban $\mathrm{C}$, Dravgan $\mathrm{S}$. The relationship between inflammatory and oxidative stress biomarkers, atherosclerosis and rheumatic disease. Curr Pharm Des. 2014;20:585-600.

13. Ridker PM. Anti-inflammatory therapy for atherosclerosis: interpreting divergent results from the CANTOS and CIRT clinical trials. J Intern Med. 2019;285:503-9.

14. Khan R. Spagnoli V, Tardif JC, L'Allier PL. Novel anti-inflammatory therapies for the treatment of atherosclerosis. Atherosclerosis. 2015;240:497-509.

15. Dressler W, Stein R. Ueber den hydroxylharnstoff. Ann Chem Pharm. 1869;150(2):242-52.

16. Madaan K, Kaushik D, Verma T. Hydroxyurea: a key player in cancer chemotherapy. Expert Rev Anticancer Ther. 2012;12(1):19-29.

17. Gallaugher LD, Henry JC, Kearns PN, et al. Ribonucleotide reductase inhibitors reduce atherosclerosis in a double-injury rabbit model. Comp Med. 2009;59(6):567-72.

18. Nakashima Y, Plump AS, Raines EW, et al. ApoE-deficient mice develop lesions of all phases of atherosclerosis throughout the arterial tree. Arterioscler Thromb. 1994;14(1):133-40.

19. van Staa TP. Carr DF, O'Meara H, McCann G, Pirmohamed M. Predictors and outcomes of increases in creatine phosphokinase concentrations or rhabdomyolysis risk during statin treatment. Br J Clin Pharmacol. 2014;78:649-59.

20. Frost $P$, Berlanger $E$, Mysore $V$, et al. Mammalian target of rapamycin inhibitors induce tumor cell apoptosis in vivo primarily by inhibiting VEGF expression and angiogenesis. J Oncol. 2013;2013:897025.

21. Kaylani SZ. Xu J, Srivastava RK, et al. Rapamycin targeting mTOR and hedgehog signaling pathways blocks human rhabdomyosarcoma growth in xenograft murine model. Biochem Biophys Res Commun. 2013;435:557-61.

22. Poder TG. Erraji J, Coulibaly LP, Koffi K. Percutaneous coronary intervention with second-generation drug-eluting stent versus bare-metal stent: Systematic review and cost-benefit analysis. PLoS One. 2017;12(5):e0177476. 
23. Naranjo A. Sokka T, Descalzo MA, et al. Cardiovascular disease in patients with rheumatoid arthritis: results from the QUEST-RA study. Arthritis Res Ther. 2008;10(2):R30.

24. Zhang R. Chen $\mathrm{S}$, Zhang $\mathrm{H}$, et al. Effects of methotrexate in a rabbit model of in-stent neoatherosclerosis: an optical coherence tomography study. Sci Rep. 2016;20(6):33657.

25. Ruiter MS. Van Tiel CM, Doornbos A, et al. Stents eluting 6-mercaptopurine reduce neointima formation and inflammation while enhancing strut coverage in rabbits. PloS One. 2015;10(9):e0138459.

\section{Figures}

$A(L)$

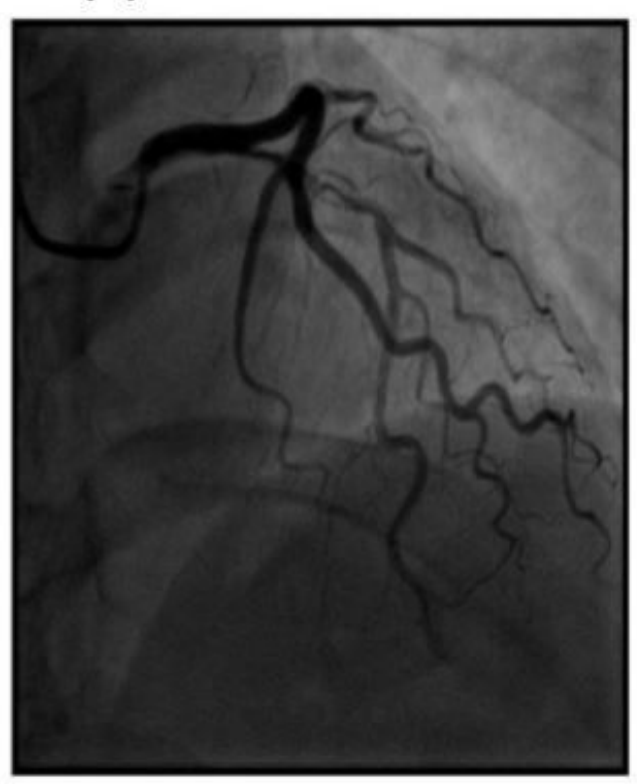

(R)

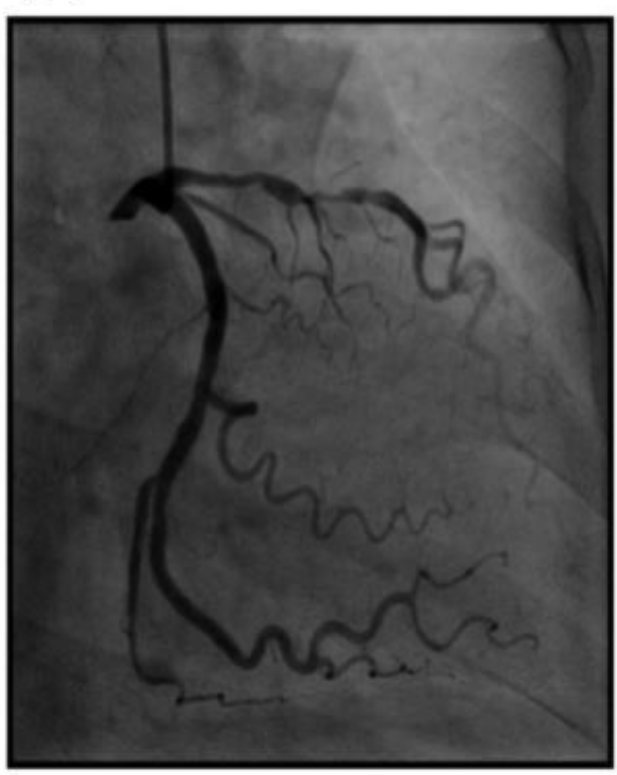

B

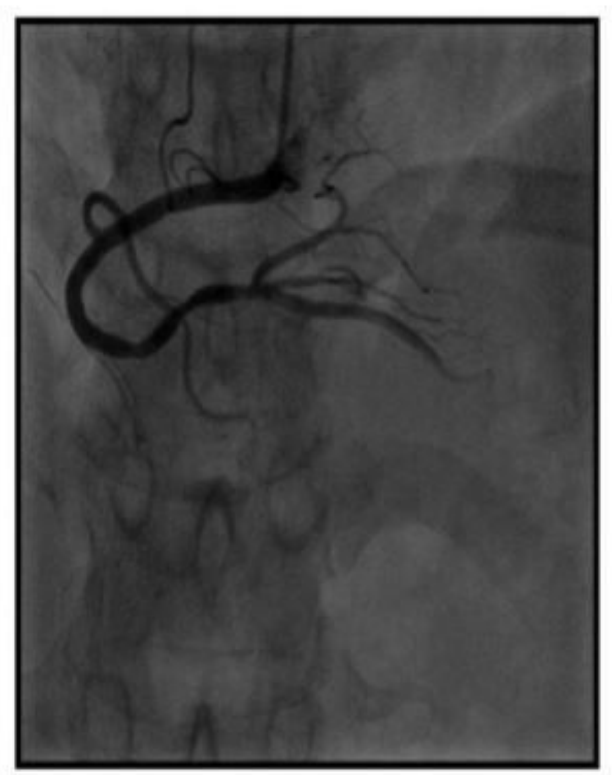

\section{Figure 1}

Result of CAG when first admitted. Severe stenosis in proximal LAD (a, L\&R), and moderate stenosis of distal RCA (b). 


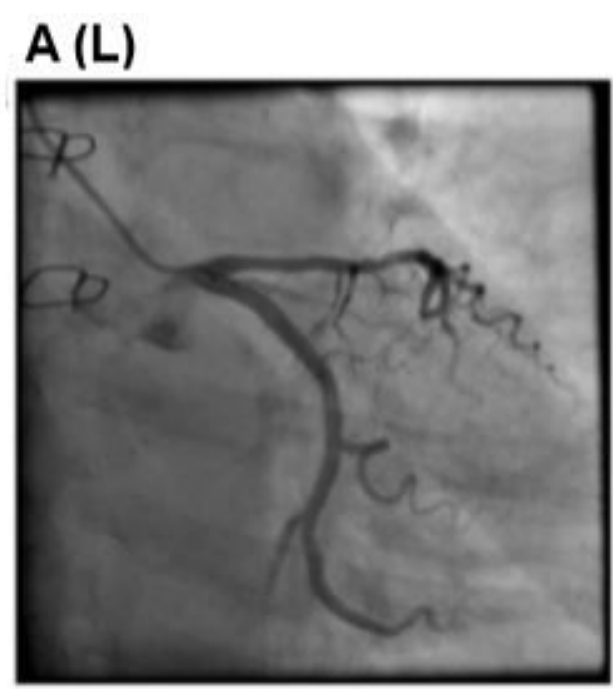

$B(L)$

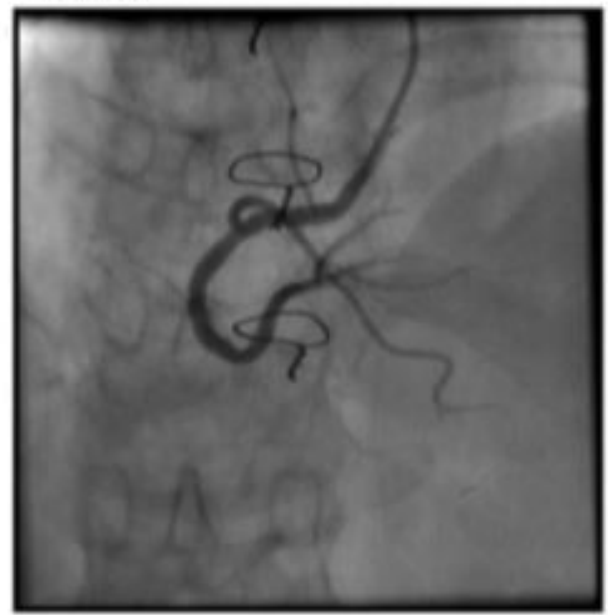

(C)

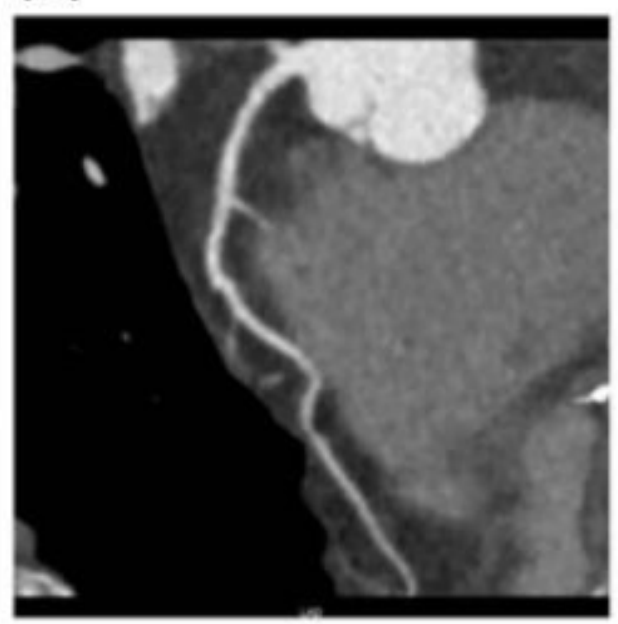

(C)

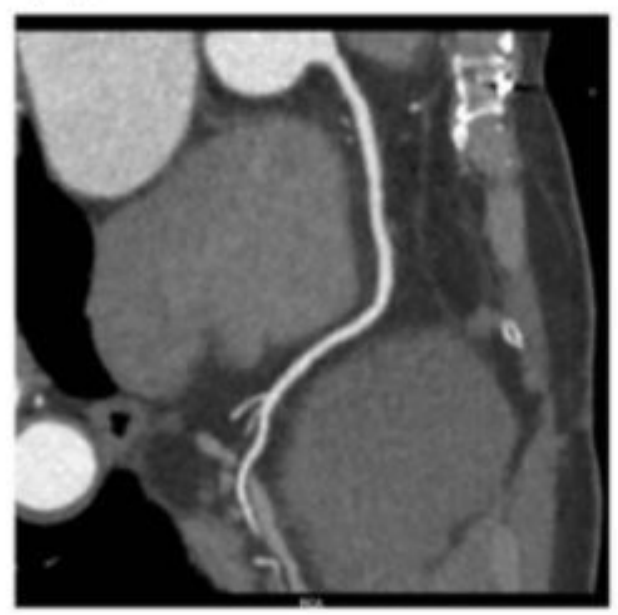

(R)

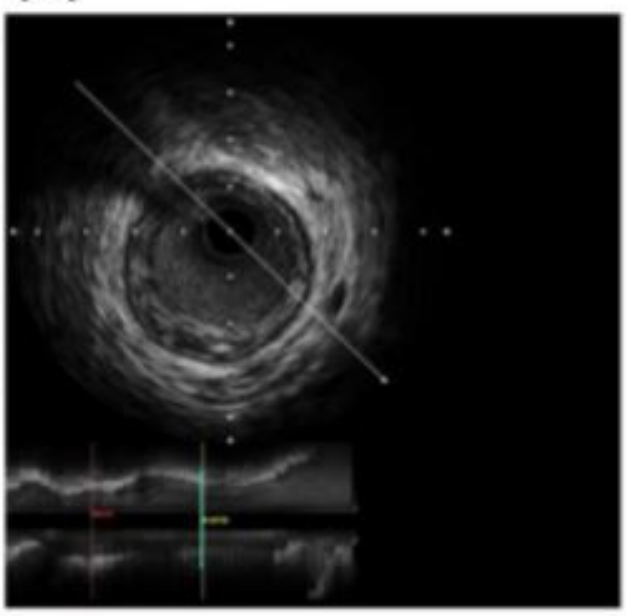

(R)

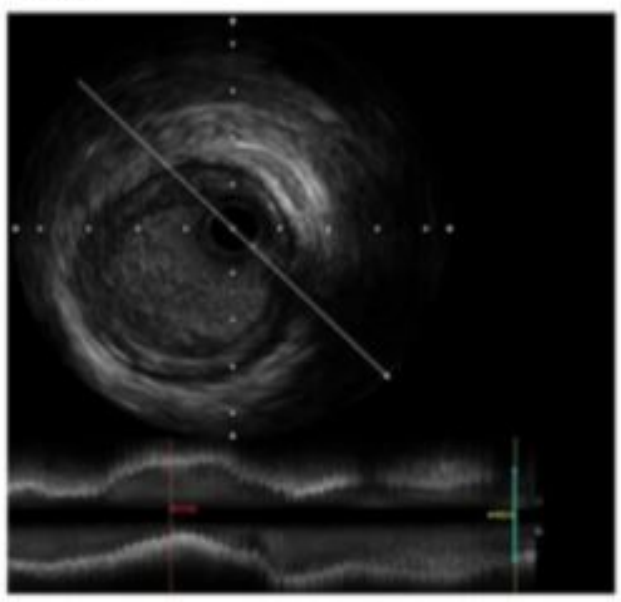

Figure 2

Result of CAG, CTA and IVUS after 3 years' follow-up. Only moderate stenosis of proximal LAD and annular fibrous plaque of proximal LAD which indicated the stability of atherosclerotic plaque (a), and no obvious stenosis of RCA was observed (b). 


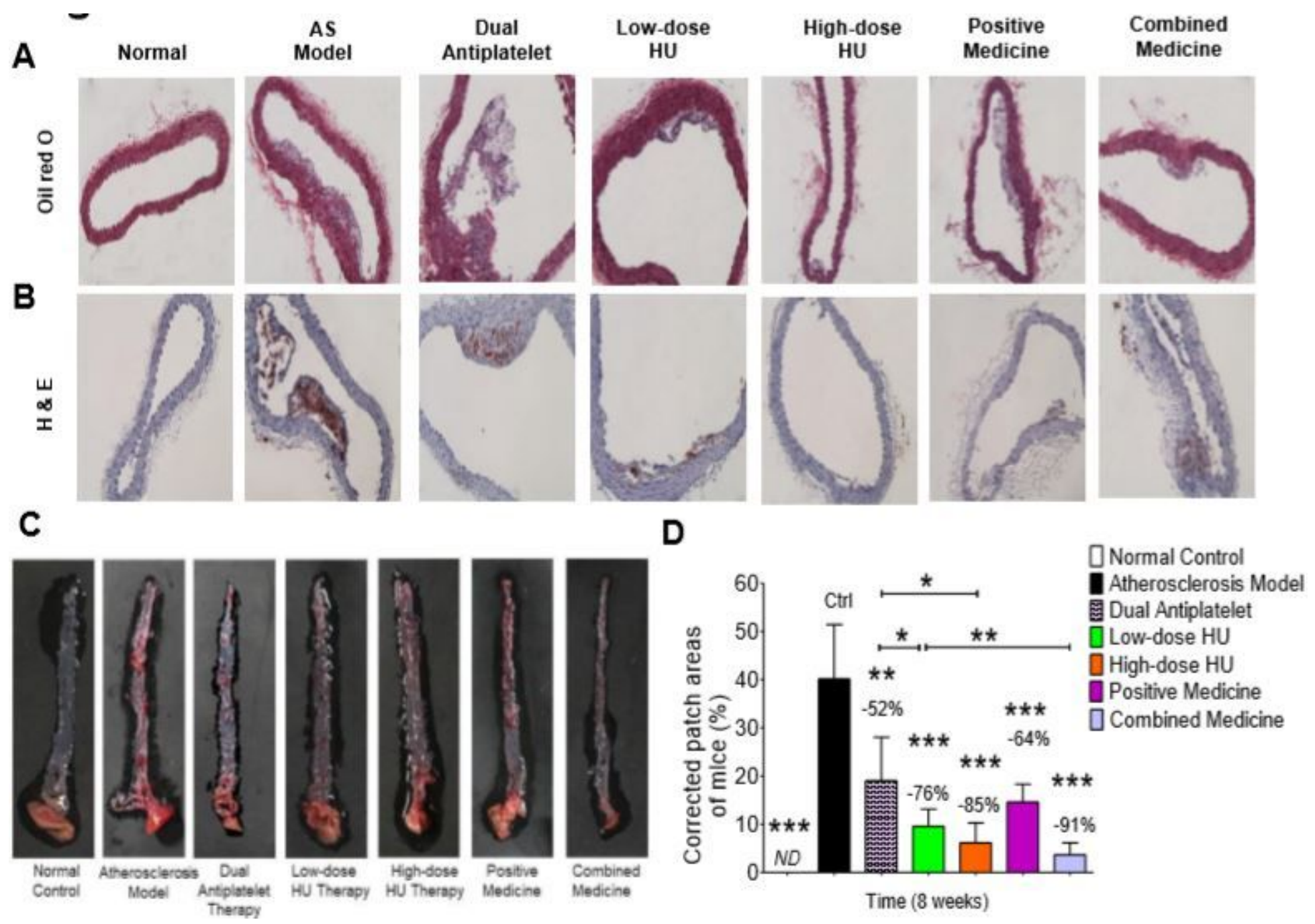

Figure 3

$\mathrm{HU}$ treatment decreased the amount and area of atherosclerotic plaques in ApoE-/- mice. (a-d) After 8 weeks of medication, atherosclerosis model group showed observable plaques compared to normal control group $(* \star \star P<0.001)$. HU therapy group presented less plaques and dosage dependent $\left({ }^{*} * \mathrm{P}<0.001\right)$. Combined medicine group present the least patch area $\left({ }^{* \star *} \mathrm{P}<0.001\right)$ and no obvious atherosclerotic plaques were observed, (a) the aortic oil red O staining, (b) H\&E staining, (c) the full-length oil red $\mathrm{O}$ staining, and (d) corrected patch areas of mice (\%). 

A
Q Normal Control
QHigh-dose HU
Atherosclerosis Model $Q$ Positive Medicine
QDual Antiplatelet
CLow-dose HU
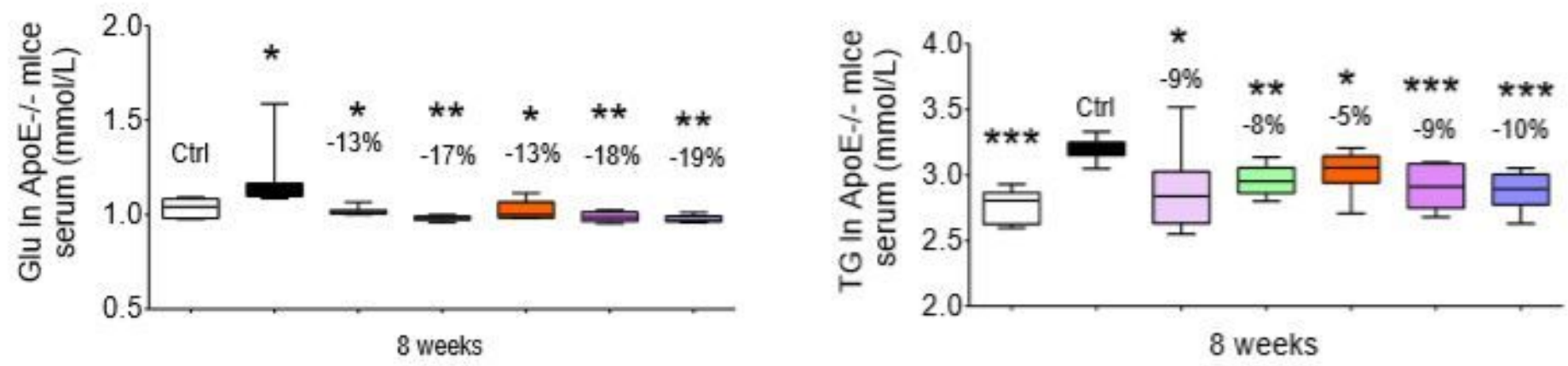

C
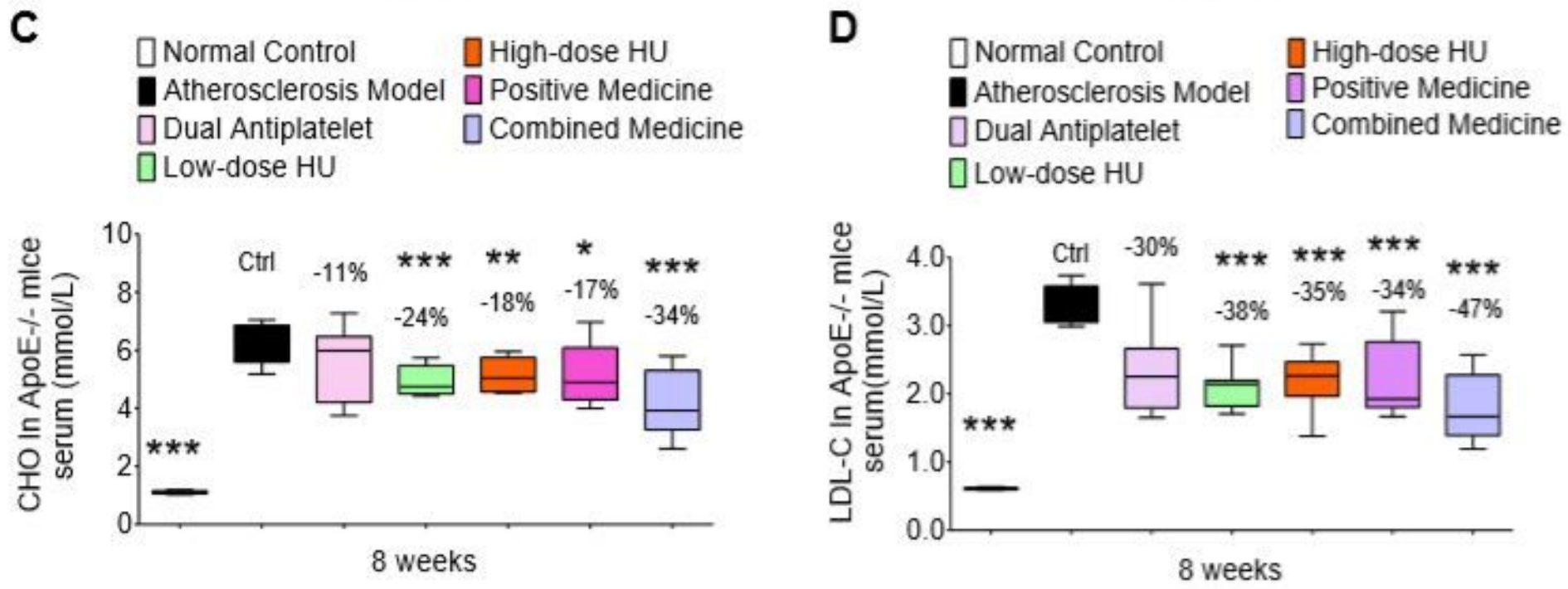

\section{Figure 4}

Glu (a), TG (b), CHO (c) and LDL-C (d) level in ApoE -/- mice serum in all the medication groups $(n=8)$. In HU therapy group, no significant variation was observed compared to the dual antiplatelet group. 
DUntreated

B

Atherosclerosis Model

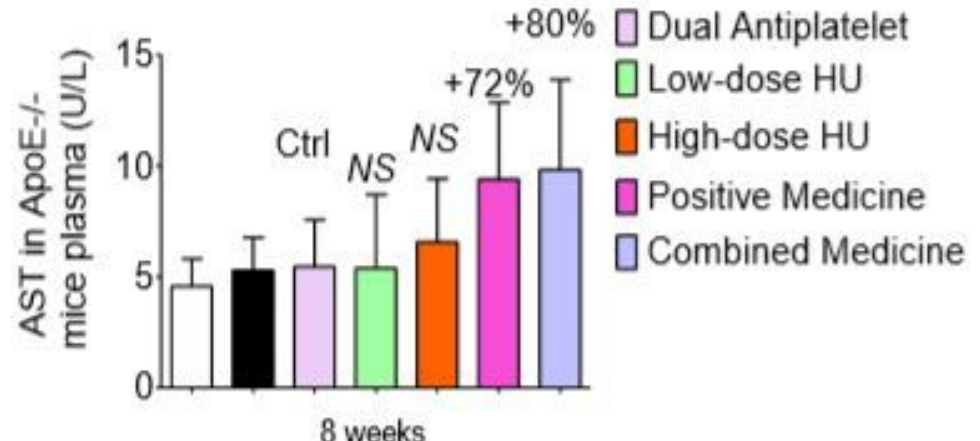

8 weeks
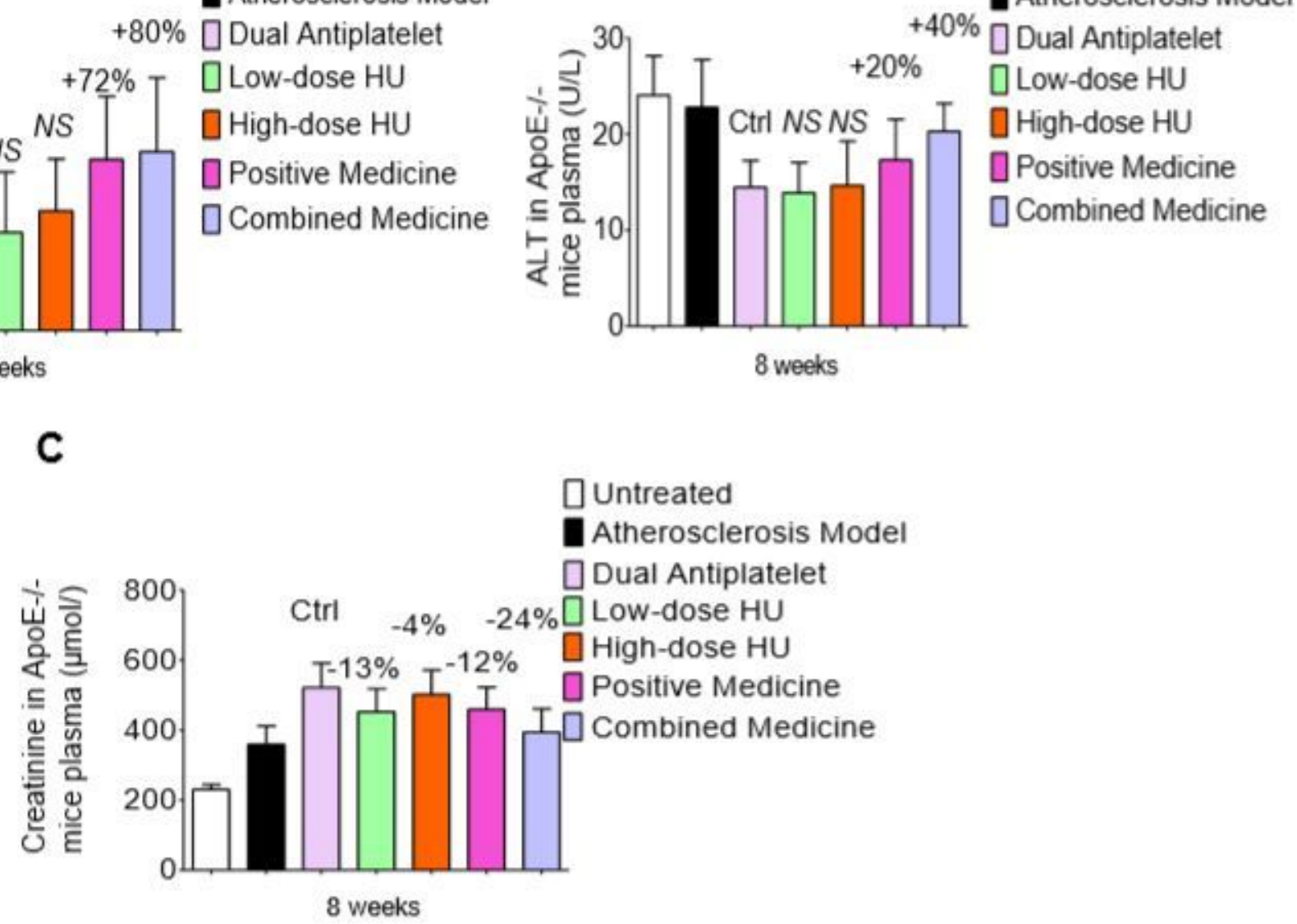

Figure 5

HU didn't increase the risk of hepatoxicity and renal toxicity in ApoE-/- mice. (a-b) AST and ALT level were significantly increased in positive medicine group ( $+40 \%$ for AST and $+72 \%$ for ALT), when no significant variation was observed between positive medicine group and combined medicine group, and (c) creatinine level were significantly increased in atherosclerosis model group $(* P<0.05)$. Among the medication groups, creatinine level was increased when no difference was observed in HU therapy group.

\section{Supplementary Files}

This is a list of supplementary files associated with this preprint. Click to download.

- ARRIVEChecklist.pdf

- Graphicalabstract.pdf 